\title{
Clinical characteristics and risk factors of sporadic Hepatitis E in central China
}

Shujun Zhang ${ }^{1 \dagger}$, Jingjing Wang ${ }^{1 \dagger}$, Quan Yuan ${ }^{2}$, Shengxiang $\mathrm{Ge}^{2}$, Jun Zhang ${ }^{2}$, Ningshao Xia ${ }^{2}$ and Deying $\operatorname{Tian}^{1^{*}}$

\begin{abstract}
Background: Epidemiological investigations, detections and vaccines of hepatitis $E$ (HE) have been paid a focus of attention in prior studies, while studies on clinical features and risk factors with a large number of sporadic $\mathrm{HE}$ patients are scarce.

Results: Sporadic HE can occur throughout the year, with the highest incidence rate in the first quarter of a year, in central of China. Of the 210 patients, $85.2 \%$ were male, and the most common clinical symptoms were jaundice (85.7\%), fatigue (70.5\%) and anorexia (64.8\%). Total bilirubin (TBil), blood urea nitrogen (BUN), and international normalized ratio (INR) were found as major risk factors for death of HE patients. There was an overall mortality of $10 \%$, and the mortality in the cirrhotic and non-cirrhotic group was $25 \%$ and $6.47 \%$, respectively. Moreover, hepatitis E virus (HEV) infected patients with liver cirrhosis had a higher mortality and incidence of complications.
\end{abstract}

Conclusions: TBil, BUN, and INR are major risk factors of mortality for HE. Liver cirrhosis can aggravate HE, and lead to a higher mortality. HEV infection can cause decompensation in patients with cirrhosis, as evidenced by a worsening Child-Pugh score.

\section{Background}

HE caused by HEV infection is transmitted by the fecaloral route and generally causes an acute self-limiting illness followed by complete recovery, which is the same as hepatitis A (HA). However, the mortality of HE is higher than $\mathrm{HA}$ and hepatitis $\mathrm{B}(\mathrm{HB})$, especially in pregnant women (with a mortality of $20 \% \sim 30 \%$ ) [1]. HE is endemic in many developing countries with poor sanitation and insufficient public-health infrastructures. Nevertheless, HEV infections are reported even in developed countries in recent years, making the disease a great threat to human health. For example, a recent study reported that a seroprevalence of HEV was found among $20 \%$ of blood donors in USA and an evidence of HEV epidemic was found in Japan[2]. HEV infections have also been documented in Australia and European Union[3-6]. Besides, cases of sporadic HE in people without histories of recent travels have been reported in developed regions.

\footnotetext{
* Correspondence: dytian@tjh.tjmu.edu.cn

+ Contributed equally

'Department of Infectious Disease, Tongji Hospital, Tongji Medical College,

Huazhong University of Science and Technology, Wuhan 430030, China
}

Full list of author information is available at the end of the article
The incidence of HE is higher and higher, while mortalities in different areas are distinct. A study in India revealed that the mortality of out-break of HE was $0.07 \%-0.6 \%[7]$. The mortality of in-hospital patients with acute HE had a mortality of $1 \% \sim 3 \%[1]$. Till now, most studies were focused on the epidemiological investigation, detections and vaccines, while studies on clinical features and risk factors of death for HE with a large number of patients are lacking. There are studies on the outcome of HEV infection in patients with chronic liver disease from India, Nepal, France and the UK[8-11]. It was showed that the mortality of HEV in patients with cirrhosis was $70 \%$ at 1 year[8]. However, similar reports from China are scarce.

\section{Methods}

\subsection{Patients}

This study included 210 in-hospital HE patients from Department of Infectious Disease of Wuhan Tongji Hospital from January 2007 to December 2008. HE case definition: alanine aminotransferase, ALT $\geqq 2.5 \times$ upper limits of normal (ULN) and HEV IgM positive, or a rising HEV IgG or HEV PCR positive[11]. Cirrhotic patients with sepsis, primary liver cancer, surgical 
obstructive jaundice, hepatorenal syndrome and those consuming alcoholic during previous 6 months were excluded from the study. The cirrhosis groups were matched for Child-Pugh score twice: the first time was 1 month before admission and the second was after admission. Each patient after discharge from the hospital was followed up 4 weekly at least for 6 months.

\subsection{Methods}

\subsubsection{Pathogenic Detection}

Sera from each patient was tested for HEV-IgM, HEVIgG, HAV-IgM, anti-HCV, HBsAg, HBsAb, HBeAg, HBeAb, HBAb using commercial ELISA kit ( Beijing Wantai Company).

\subsubsection{Reverse transcription and nested PCR for HEV[12]}

HEV RNA was extracted and precipitated from $200 \mu \mathrm{l}$ of serum samples by acid-guanidinium-phenol method (Trizol LS Reagent Invitrogen, USA). Reverse primer E5: 5'CTACACGAAACCGARAGW ( $\mathrm{R}=\mathrm{A}$ or $\mathrm{G}, \mathrm{W}=\mathrm{A}$ or C) was used to reverse transcription. With primer E1 (5'CTGTTTAAYCTTGCTGACAC 3' $(\mathrm{Y}=\mathrm{C}$ or T)) and primer E5, the first round of amplification was completed $\left(94^{\circ} \mathrm{C}\right.$ pre-degeneration for $5 \mathrm{~min}, 94^{\circ} \mathrm{C}$ for $40 \mathrm{~s}$, $53^{\circ} \mathrm{C}$ for $40 \mathrm{~s}, 72^{\circ} \mathrm{C}$ for 40 s, followed by 35 cycles, $72^{\circ} \mathrm{C}$ for $10 \mathrm{~min}$ ). The amplified material was used for the second-round nested amplification with primers E2 (5'GACAGAATTGATTTCGTCG 3') and E4 (5'GTCC TAATACTRTTGGTTGT3' ( $\mathrm{R}=\mathrm{A}$ or $\mathrm{G})$ ). The length of PCR product corresponding to ORF2 sequence was 189 bp (6298nt-6486nt).

\subsubsection{Biochemical Tests}

An automatic biochemical analyzer (Beckman L220) was used to analyze biochemical parameters, such as ALT, BUN, TBil, INR, aspartate aminotransferase (AST), albumin (ALB), total cholesterol (Tchol), lactate dehydrogenase $(\mathrm{LDH})$, creatinine $(\mathrm{Cr})$, prothrombin activity (PTA).

\subsubsection{Diagnosis of cirrhosis}

Forty of 210 patients were diagnosed with liver cirrhosis before HEV infection, which was established by conventional clinical, biochemical, imaging, and endoscopic criteria[8]. The etiology of cirrhosis in patients was HB 20, Schistosomiasis 7, alcohol 3, hepatitis C 1, autoimmune hepatitis 1, alcohol plus HB 5, HB plus Schistosomiasis 1, Schistosomiasis plus alcohol 1, and HB plus Schistosomiasis and alcohol 1 case.

\subsubsection{Statistics}

Quantitative variables were expressed as means $( \pm \mathrm{SD})$ and compared by the Student t-test, or represented as median (25th percentile-75th percentile). Mann-Whitney $\mathrm{U}$ test was used to compare serum biochemical indicators and $\chi 2$ or Fisher's exact test was used to enumeration data. Odds ratio (OR) for all variables was calculated by univariate and multivariate logistic regression. All statistical calculations were performed using SPSS software 13.0.

\section{Results}

\subsection{Etiology Detected Results}

Among 210 patients, 125 were diagnosed being infected with HEV alone, 1 co-infected with hepatitis A virus (HAV), and 75 co-infected with hepatitis B virus (HBV), 2 with hepatitis $\mathrm{C}$ virus (HCV), 3 with cytomegalovirus (CMV), 3 with Epstein-Barr virus (EBV), 1 with CMV + EBV. Forty of the 210 patients had liver cirrhosis as well. Seventy-eight patients had detectable HEV RNA in their sera. All of them were genotype 4 which were confirmed by bidirectional sequencing and phylogenetic.

\subsection{The Incidence Rate of HE in Different Seasons}

It was found that 122 and 88 patients were infected with HE in 2007 and 2008, respectively. Although patients could be infected throughout a year, the incidence rate of $\mathrm{HE}$ was highest in the first quarter (from January to March) (Figure 1).

\subsection{The Distribution of HE patients' Gender and Age}

Of the 210 patients, 179 cases were male $(85.2 \%)$ and 31 cases were female $(14.8 \%)$. The ratio of male to female was approximately $5.8: 1$. Ages of the patients ranged from 17 to 92 years old $(48.7 \pm 14.9)$. There was only 1 case under 20 years old $(0.5 \%), 90 \%$ of the patients were over 30 years of age (Figure 2).

\subsection{Clinical Symptoms and Serum Biochemistry}

Jaundice, fatigue and anorexia were the most common clinical symptoms, with incidence rates of $85.7 \%$ (180/ 210), $70.5 \%$ (148/210), and 64.7\% (136/210), respectively. Other less common symptoms were also observed,

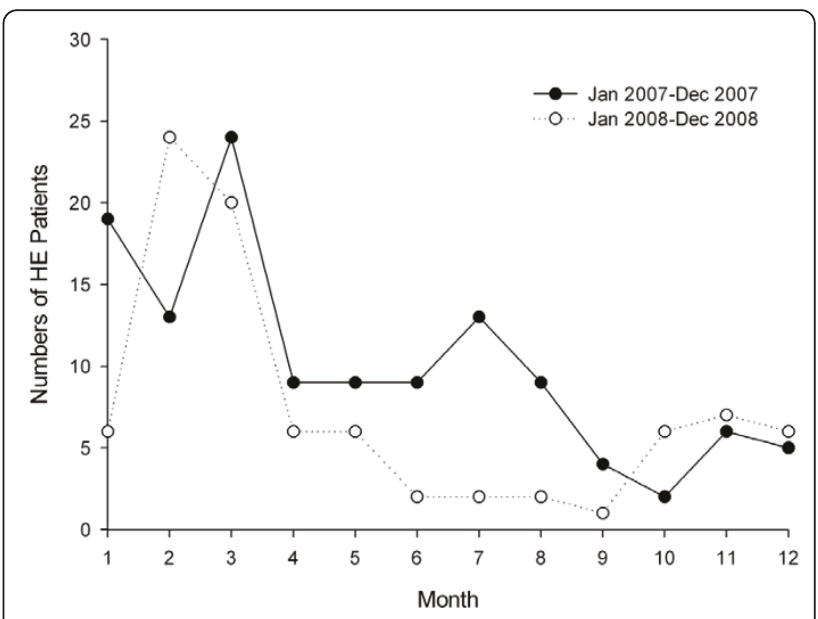

Figure 1 The incidence of HE infection in different months of the year. 


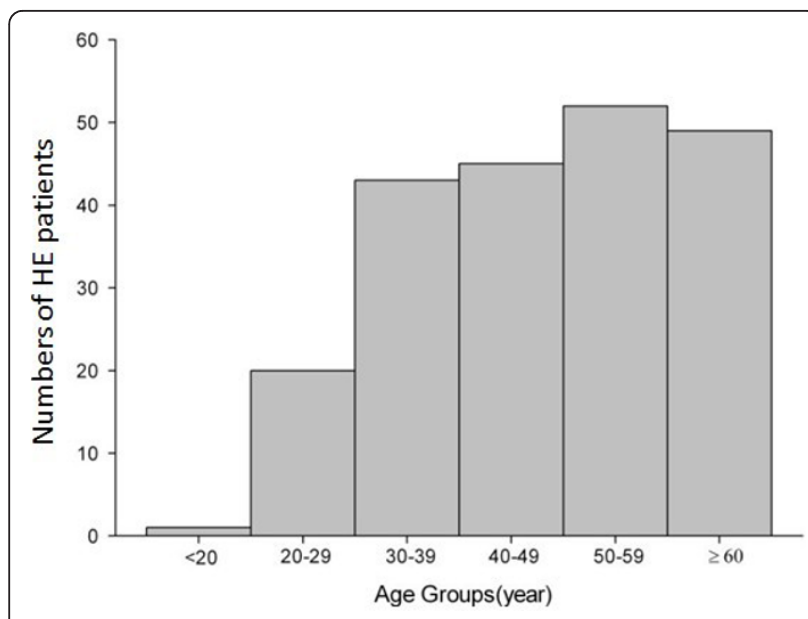

Figure 2 Number of HE patients according to age group

including abdominal distention (55/210, 26.2\%), nausea $(23 / 210,11.0 \%)$, vomiting $(14 / 210,6.7 \%)$ and fever (16/ 210. $7.6 \%)$.

All patients were followed up for six months. Twenty-one patients died during this period, for a mortality of $10 \%$ (95\% CI: $6.63 \%-14.80 \%$ ). The main causes of death were upper gastrointestinal bleeding $(6 / 21)$, hepatorenal syndrome $(12 / 21)$, and hepatic encephalopathy $(3 / 21)$. The results of clinical features and serum biochemistry were summarized in Table 1. Patients with old ages and liver cirrhosis were more likely to die than others. The level of TBil, Tchol, LDH, PTA and INR were significantly different between died patients and survivors. BUN and $\mathrm{Cr}$, which reflect the renal function, were higher in the deceased group than in the survivor group.

From table 2 we can see that age $(\mathrm{OR}=1.067)$, TBil $(\mathrm{OR}=1.010)$, ALB $(\mathrm{OR}=0.781)$, Tchol $(\mathrm{OR}=0.164)$, $\mathrm{LDH}(\mathrm{OR}=1.007), \mathrm{BUN}(\mathrm{OR}=1.299), \mathrm{Cr}(\mathrm{OR}=$ 1.010), PTA ( $\mathrm{OR}=0.956)$, and INR $(\mathrm{OR}=6.113)$ were the death risk factors of HE. Multivariate analysis of death risk factors in patients with HE were shown in Table 3. TBil, BUN, and INR were major risk factors

\subsection{The Influence of Liver Cirrhosis on the Prognosis of Hepatitis E}

Compared with hepatitis E infection patients without liver cirrhosis, the mortality of those with liver cirrhosis was increased ( $25 \%$ vs. $6.47 \%, P=0.002)$. The incidence of hepatic encephalopathy (6/40 vs. $8 / 170, \mathrm{p}=0.03)$, hepatorenal syndrome $(8 / 40$ vs. $5 / 170, P<0.001)$ and spontaneous bacterial peritonitis $(24 / 40$ vs. $14 / 170, P<$ 0.001 ) were higher than HEV infected patients without cirrhosis. The difference of ALT, GGT, ALB, Tchol, PTA, INR, BUN between two groups was significant as well (table 4).
Table 1 Comparison of clinical and biochemical parameters between the deceased group and the survival group

\begin{tabular}{|c|c|c|c|}
\hline Variable & Deceased group & Survival group & $\begin{array}{l}P \\
\text { value }\end{array}$ \\
\hline $\begin{array}{l}\text { Number of } \\
\text { patients }\end{array}$ & 21 & 189 & - \\
\hline Age in years & $59.67 \pm 13.99$ & $47.22 \pm 13.97$ & 0.0002 \\
\hline $\begin{array}{l}\text { Gender(male/ } \\
\text { female) }\end{array}$ & $20 / 1$ & $159 / 30$ & NS \\
\hline $\mathrm{ALT}(\mathrm{U} / \mathrm{L})$ & $\begin{array}{l}731.00(202.00- \\
1910.50)\end{array}$ & $\begin{array}{l}1070.50(381.00- \\
1773.00)\end{array}$ & NS \\
\hline AST(U/L) & $\begin{array}{l}659.00(228.10- \\
1386.00)\end{array}$ & $\begin{array}{l}551.00(189.00- \\
1242.25)\end{array}$ & NS \\
\hline GGT(U/L) & $62.00(44.00-115.50)$ & $131.00(73.00-221.00)$ & 0.007 \\
\hline TBil $(\mu \mathrm{mol} / \mathrm{L})$ & $\begin{array}{l}551.40(409.35- \\
607.95)\end{array}$ & $163.81(69.83-275.90)$ & $\begin{array}{l}< \\
0.001\end{array}$ \\
\hline$A L B(g / L)$ & $29.60(26.40-31.30)$ & $34.15(31.15-37.78)$ & $\begin{array}{l}< \\
0.001\end{array}$ \\
\hline$A L P(U / L)$ & $\begin{array}{l}199.00(144.50- \\
241.50)\end{array}$ & $\begin{array}{l}170.00(131.00- \\
218.00)\end{array}$ & NS \\
\hline Tchol(mmol/L) & $1.33(0.98-1.86)$ & $3.23(2.51-3.92)$ & $\begin{array}{l}< \\
0.001\end{array}$ \\
\hline $\mathrm{LDH}(\mathrm{U} / \mathrm{L})$ & $\begin{array}{l}274.00(223.00- \\
385.00)\end{array}$ & $\begin{array}{l}200.00(151.00- \\
261.00)\end{array}$ & 0.001 \\
\hline $\mathrm{CHE}(\mathrm{U} / \mathrm{L})$ & $\begin{array}{l}2941.00(2060.00- \\
4269.50)\end{array}$ & $\begin{array}{l}4454.50(3021.25- \\
5808.75)\end{array}$ & 0.003 \\
\hline $\mathrm{BUN}(\mathrm{mmol} / \mathrm{L})$ & $14.50(6.07-24.51)$ & $4.44(3.64-5.61)$ & $\begin{array}{l}< \\
0.001\end{array}$ \\
\hline $\mathrm{Cr}(\mu \mathrm{mol} / \mathrm{L})$ & $124.05(70.83-253.55)$ & $64.00(55.38-73.78)$ & $\begin{array}{l}< \\
0.001\end{array}$ \\
\hline PTA(\%) & $46.00(32.00-70.00)$ & $92,50(71.00-112.75)$ & $\begin{array}{l}< \\
0.001\end{array}$ \\
\hline INR & $1.66(1.35-2.19)$ & $1.14(1.05-1.30)$ & $\begin{array}{l}< \\
0.001\end{array}$ \\
\hline
\end{tabular}

ALT, alanine aminotransferase; AST, aspartate aminotransferase; GGT, $\gamma$ glutamyltransferase; TBil, total bilirubin; ALB, albumin; ALP, alkaline phosphatase; Tchol, total cholesterol; $\mathrm{LDH}$, lactate dehydrogenase; CHE, cholinesterase; BUN, blood urea nitrogen; $\mathrm{Cr}$, creatinine; PTA, prothrombin activity; INR, international normalized ratio; and NS, not significant.

The 40 HE patients with cirrhosis were assessed by Child-Pugh's score on the basis of reviewing their history, clinical symptoms and laboratory data one month ago. And this was before presenting HEV infection. Thirty-two cases (80\%) were at the Child-Pugh stage A, 7 cases $(17.5 \%)$ at the Child-Pugh stage $B$, and 1 case $(2.5 \%)$ at the Child-Pugh stage $C$, with a mean score of $5.85 \pm 1.29$. After being in hospital, 4 cases $(10 \%)$ were at stage A, 21 cases $(52.5 \%)$ at stage B, and 15 cases $(37.5 \%)$ at stage $C$, with a mean score of $8.83 \pm 1.97$. Child-Pugh's score of the patients after being in hospital was significantly worse than the patients before HEV infection $(5.85 \pm 1.29$ vs. $8.83 \pm 1.97, P<0.001)$.

\section{Discussion}

There are four genotypes of HEV, but only one serotype. Genotypes 1 and 2 exclusively infect humans, 
Table 2 Risk factors of mortality among patients with HE by Univariate analysis

\begin{tabular}{|c|c|c|c|c|}
\hline Variable & Categories & Odds Ratio & $95 \% \mathrm{Cl}$ & $P$ value \\
\hline Age & Continuous & 1.067 & $1.029-1.106$ & 0.0005 \\
\hline \multirow[t]{2}{*}{ Gender } & Female & 1 & & \\
\hline & Male & 4.173 & $0.538-32.343$ & NS \\
\hline$A L T$ & Continuous & 1.000 & 0.999-1.000 & NS \\
\hline AST & Continuous & 1.000 & $1.000-1.000$ & NS \\
\hline GGT & Continuous & 1.000 & 0.997-1.002 & NS \\
\hline TBil & Continuous & 1.010 & 1.006-1.013 & $<0.001$ \\
\hline ALB & Continuous & 0.781 & 0.696-0.877 & $<0.001$ \\
\hline ALP & Continuous & 1.001 & 0.999-1.003 & NS \\
\hline Tchol & Continuous & 0.164 & $0.079-0.340$ & $<0.001$ \\
\hline $\mathrm{LDH}$ & Continuous & 1.007 & 1.003-1.011 & 0.001 \\
\hline $\mathrm{CHE}$ & Continuous & 1.000 & 0.999-1.000 & 0.005 \\
\hline BUN & Continuous & 1.299 & $1.149-1.469$ & 0.001 \\
\hline $\mathrm{Cr}$ & Continuous & 1.010 & 1.003-1.016 & 0.003 \\
\hline PTA & Continuous & 0.956 & 0.937-0.975 & $<0.001$ \\
\hline INR & Continuous & 6.113 & $2.401-15.561$ & $<0.001$ \\
\hline
\end{tabular}

ALT, alanine aminotransferase; AST, aspartate aminotransferase; GGT, $\gamma$ glutamyltransferase; TBil, total bilirubin; ALB, albumin; ALP, alkaline phosphatase; Tchol, total cholesterol; $\mathrm{LDH}$, lactate dehydrogenase; CHE, cholinesterase; BUN, blood urea nitrogen; $\mathrm{Cr}$, creatinine; PTA, prothrombin activity; INR, international normalized ratio; and NS, not significant.

whereas genotypes 3 and 4 can also infect other animals, particularly pigs. Genotype 4 has been proven the dominant genotype in China since the year of 2000[13-16]. In this study, seventy eight (37.14\%) of 210 patients had detectable HEV RNA in their sera. All patients in whom HEV RNA was isolated were infected with genotype 4, which was congruent with those from China.

Contaminated drinking water has been served as sources of several outbreaks of HE in India[17-19]. It demonstrates that the contaminated water is one important reason for outbreak of $\mathrm{HE}$, therefore outbreaks of $\mathrm{HE}$ were found in rainy or floodwater seasons. However, in contrast with the outbreak of $\mathrm{HE}$, our data illustrated that the sporadic HE infection could occur throughout a year, and exhibited obvious seasonal occurrence. The incidence of HE in the first quarter of 2007 and 2008 were much higher than that in other quarters. HE is a zoonotic disease. The strongest evidence of zoonotic transmission of HE is from Japan[20,21]. Similarly, more meat in central of China is consumed in the first quarter

Table 3 Risk factors of mortality among patients with HE by Multivariate analysis

\begin{tabular}{lllll}
\hline Variables & Categories & Odds Ratio & $\mathbf{9 5 \%} \mathbf{C l}$ & $\boldsymbol{P}$ value \\
\hline TBil & Continuous & 1.0009 & $1.004-1.014$ & $<0.001$ \\
BUN & Continuous & 1.178 & $1.034-1.341$ & 0.014 \\
INR & Continuous & 9.216 & $1.969-43.129$ & 0.005 \\
\hline
\end{tabular}

TBil, total bilirubin; BUN, blood urea nitrogen; INR, international normalized ratio. of a year, since people in China celebrate their most important traditional festivals (the Spring Festival and the Lantern Festival) in the first quarter. So, this may be a possible reason for the seasonal occurrence. Another reason is that the weather in central of China in the first quarter is conducive to virus multiplication and propagation.

Although HEV and HAV are similar viruses in terms of the transmission mode and clinical manifestation, $90 \%$ of the HE patients were over 30 years of age, and we saw only one case under age 20 in this study, which are rather different from the HA patients. The reason for the difference of the age distribution is could be that HEV has a much lower secondary attack rate among exposed household members compared with the stable HAV (with a secondary attack rate of 20\%-50\%) [22,23].

Another noticeable feature of $\mathrm{HE}$ is that the male patients were much more than the female patients (5.8:1). The result is in accordance with other reports. For example, a nationwide survey of the prevalence of IgG anti-HEV in qualified blood donors throughout Japan showed that prevalence of IgG anti-HEV was higher in men $(3.9 \%)$ than in women $(2.9 \%)$ [24]. Similar results were also reported in Bangladeshi and Taiwan $[25,26]$. In this study, jaundice, fatigue and anorexia were most common clinical manifestations. The mortality was $10.0 \%$ in our study, higher than the previously reported rate of $1 \%$ to $3 \%$. This difference may be mainly due to the sample selection in this study: a considerable proportion of patients whom were hospital referraled from municipal hospitals were critically ill, leading to a high mortality in the overall sample (Tongji hospital is the largest hospital in the middle part of China).

The multivariate analysis showed that TBil, BUN, and INR were major risk factors of mortality for $\mathrm{HE}$, which was useful to assess the prognosis of HE. Infection of $\mathrm{HEV}$ in patients on the base of chronic liver diseases could make the chronic liver disease more severe, and cause decomposition and death[27]. Patients with cirrhosis were prone to infect $\mathrm{HEV}$, and the mortality of $\mathrm{HEV}$ infected cirrhotics at 4 weeks and 12 month was increased compared with that of non-infected cirrhotics [8]. Our study also showed that the mortality of HEV infected patients with cirrhosis was higher. And the incidence of hepatic encephalopathy, hepatorenal syndrome and spontaneous bacterial peritonitis were higher than $\mathrm{HEV}$ infected patients. It reveals that patients with cirrhosis would have a worse condition as well as a poor prognosis. The hepatic reserve function of cirrhotics deteriorated after HEV infection.

In conclusion, we found that TBil, BUN, and INR were major risk factors of mortality of HE based on 210 patients in central of China. Liver cirrhosis could make 
Table 4 Comparison of demographic, biochemical parameters as well as Complications and mortality frequencies among patients infected with genotype $4 \mathrm{HEV}$ with and without liver cirrhosis

\begin{tabular}{llll}
\hline Parameter & HEV & & P value \\
\cline { 2 - 4 } & with cirrhosis & Without cirrhosis & - \\
\hline Number of patients & 40 & 170 & NS \\
Age in years & $51.78 \pm 14.70$ & $48.02 \pm 14.55$ & $\mathrm{NS}$ \\
Gender(male/female) & $36 / 4$ & $143 / 27$ & 0.002 \\
Mortality & $10(25 \%)$ & $11(6.47 \%)$ & $<0.001$ \\
Complications & & & $\mathrm{NS}$ \\
Spontaneous peritonitis & $24(60 \%)$ & $14(8.24 \%)$ & $<0.001$ \\
Upper gastrointestinal hemorrhage & $2(5 \%)$ & $4(2.35 \%)$ & 0.03 \\
Hepatorenal syndrome & $8(20 \%)$ & $5(2.94 \%)$ & 0.02 \\
Hepatic encephalopathy & $6(15 \%)$ & $8(4.71 \%)$ & $\mathrm{NS}$ \\
ALT(U/L) & $574.5(150.25-1612.25)$ & $1158(478.75-1816.75)$ & 0.002 \\
AST(U/L) & $310.5(117-1198.5)$ & $670.5(208-1265.2)$ & $\mathrm{NS}$ \\
GGT(U/L) & $85(52.5-114.75)$ & $131(67-229)$ & $<0.001$ \\
TBil(umol/L) & $242.6(115.6-530.3)$ & $197.93(82.6-298.85)$ & $<0.001$ \\
ALB(g/L) & $31(27.65-32.98)$ & $34.1(31.58-37.8)$ & 0.001 \\
Tchol(mmol/L) & $2.17(1.71-3.27)$ & $3.18(2.45-3.9)$ & 0.032 \\
CHE(U/L) & $3014.5(2125.75-3967)$ & $4356(3039.25-5752.25)$ & $<$ \\
BUN (mmol/L) & $5.5(4-9.29)$ & $4.65(3.64-6)$ & $<0.001$ \\
Cr(umol/L) & $64.9(55.35-116.6)$ & $65.5(56.2-78.8)$ & $<0.001$ \\
PTA(\%) & $63(42-85)$ & $93(72.95-113.5)$ & \\
INR & $1.41(1.19-1.75)$ & $1.13(1.04-1.29)$ & \\
\hline
\end{tabular}

HEV, hepatitisE virus; ALT, alanine aminotransferase; AST, aspartate aminotransferase; GGT, $\gamma$-glutamyltransferase; TBil, total bilirubin; ALB, albumin; ALP, alkaline phosphatase; Tchol, total cholesterol; LDH, lactate dehydrogenase; CHE, cholinesterase; BUN, blood urea nitrogen; $\mathrm{Cr}$, creatinine; PTA, prothrombin activity; INR, international normalized ratio; and NS, not significant

the HE more severe, and result in a higher mortality. And HEV infection could cause decompensation in patients with cirrhosis. Although the pathogenesis of $\mathrm{HE}$ has not been clarified due to the lack of effective cell or animal models of HEV infection, the results are helpful to provide us some ideas for clinical diagnosis, treatment and basic research of HE.

\section{Acknowledgements}

The authors are grateful to P Yin, professor, Public Health College, Huazhong University of Science and Technology, for his help in the statistical analysis of this study.

\section{Author details}

${ }^{1}$ Department of Infectious Disease, Tongji Hospital, Tongji Medical College, Huazhong University of Science and Technology, Wuhan 430030, China. ${ }^{2}$ National Institute of Diagnostics and Vaccine Development of Infectious Diseases, Xiamen University, Xiamen 361005, China.

\section{Authors' contributions}

SJZ conceived of the study, and participated in its design and coordination and helped to draft the manuscript. JJW participated in collecting samples and following up. QY and SXG carried out the molecular genetic studies, participated in the sequence alignment. JZ performed the statistical analysis. NSX and DYT participated in the design of the study. All authors read and approved the final manuscript.

\section{Competing interests}

The authors declare that they have no competing interests.
Received: 19 January 2011 Accepted: 1 April 2011

Published: 1 April 2011

\section{References}

1. Emerson SU, Purcell RH: Hepatitis E virus. Rev Med Virol 2003, 13: 145-154.

2. Emerson SU, Purcell RH: Running like water-the omnipresence of hepatitis E. N Engl J Med 2004, 351: 2367-2368.

3. Moaven L, Van Asten M, Crofts N, Locarnini SA: Seroepidemiology of hepatitis E in selected Australian populations. J Med Virol 1995, 45: 326-330

4. Krawczynski K, Aggarwal R, Kamili S: Hepatitis E. Infect Dis Clin North Am 2000, 14: 669-687.

5. Grieco A, Miele L, Gasbarrini G, Grillo R: Sporadic HEV hepatitis in Italy. Gut 2001, 48: 580

6. Coursaget $P$, Depril N, Buisson $Y$, Molinie C, Roue R: Hepatitis type E in a French population: detection of anti-HEV by a synthetic peptide-based enzyme-linked immunosorbent assay. Res Virol 1994, 145: 51-57.

7. Naik SR, Aggarwal R, Salunke PN, Mehrotra NN: A large waterborne viral hepatitis E epidemic in Kanpur, India. Bull World Health Organ 1992, 70: 597-604

8. Kumar Acharya S, Kumar Sharma P, Singh R, Kumar Mohanty S, Madan K, Kumar Jha J, Kumar Panda S: Hepatitis E virus (HEV) infection in patients with cirrhosis is associated with rapid decompensation and death. $J$ Hepatol 2007, 46: 387-394.

9. Kc S, Sharma D, Basnet BK, Mishra AK: Effect of acute hepatitis E infection in patients with liver cirrhosis. JNMA J Nepal Med Assoc 2009, 48: 226-229.

10. Peron JM, Bureau C, Poirson H, Mansuy JM, Alric L, Selves J, Dupuis E, Izopet J, Vinel JP: Fulminant liver failure from acute autochthonous hepatitis $E$ in France: description of seven patients with acute hepatitis E and encephalopathy. J Viral Hepat 2007, 14: 298-303.

11. Dalton HR, Hazeldine S, Banks M, ljaz S, Bendall R: Locally acquired hepatitis E in chronic liver disease. Lancet 2007, 369: 1260. 
12. Zhang S, Tian D, Zhang Z, Xiong J, Yuan Q, Ge S, Zhang J, Xia N: Clinical significance of anti-HEV IgA in diagnosis of acute genotype 4 hepatitis $E$ virus infection negative for anti-HEV IgM. Dig Dis Sci 2009, 54: 2512-2518.

13. Yu Y, Sun J, Liu M, Xia L, Zhao C, Harrison TJ, Wang Y: Seroepidemiology and genetic characterization of hepatitis $\mathrm{E}$ virus in the northeast of China. Infect Genet Evol 2009, 9: 554-561.

14. Liu Z, Chi B, Takahashi K, Mishiro S: A genotype IV hepatitis E virus strain that may be indigenous to Changchun, China. Intervirology 2003, 46 252-256.

15. Li RC, Ge SX, Li YP, Zheng YJ, Nong Y, Guo QS, Zhang J, Ng MH, Xia NS: Seroprevalence of hepatitis E virus infection, rural southern People's Republic of China. Emerg Infect Dis 2006, 12: 1682-1688.

16. Dong C, Dai X, Shao JS, Hu K, Meng JH: Identification of genetic diversity of hepatitis $E$ virus (HEV) and determination of the seroprevalence of HEV in eastern China. Arch Virol 2007, 152: 739-746.

17. Swain SK, Baral P, Hutin YJ, Rao TV, Murhekar M, Gupte MD: A hepatitis E outbreak caused by a temporary interruption in a municipal water treatment system, Baripada, Orissa, India, 2004. Trans R Soc Trop Med Hyg 2010, 104: 66-69.

18. Prinja S, Kumar S, Reddy GM, Ratho RK, Kumar R: Investigation of viral hepatitis E outbreak in a town in Haryana. J Commun Dis 2008, 40: 249-254.

19. Martolia HC, Hutin Y, Ramachandran V, Manickam P, Murhekar M, Gupte M: An outbreak of hepatitis $E$ tracked to a spring in the foothills of the Himalayas, India, 2005. Indian J Gastroenterol 2009, 28: 99-101.

20. Tamada $Y$, Yano $K$, Yatsuhashi $H$, Inoue O, Mawatari F, Ishibashi H: Consumption of wild boar linked to cases of hepatitis E. J Hepatol 2004, 40: 869-870

21. Matsuda H, Okada K, Takahashi K, Mishiro S: Severe hepatitis E virus infection after ingestion of uncooked liver from a wild boar. J Infect Dis 2003, 188: 944.

22. Rab MA, Bile MK, Mubarik MM, Asghar H, Sami Z, Siddiqi S, Dil AS, Barzgar MA, Chaudhry MA, Burney MI: Water-borne hepatitis E virus epidemic in Islamabad, Pakistan: a common source outbreak traced to the malfunction of a modern water treatment plant. Am J Trop Med Hyg 1997, 57: 151-157.

23. Koff RS: Hepatitis A. Lancet 1998, 351: 1643-1649.

24. Takeda H, Matsubayashi K, Sakata H, Sato S, Kato T, Hino S, Tadokoro K, Ikeda $\mathrm{H}$ : A nationwide survey for prevalence of hepatitis $\mathrm{E}$ virus antibody in qualified blood donors in Japan. Vox Sang 2010, 99: 307-13.

25. Labrique AB, Zaman K, Hossain Z, Saha P, Yunus M, Hossain A, Ticehurst t, Nelson KE: Population seroprevalence of hepatitis $E$ virus antibodies in rural Bangladesh. Am J Trop Med Hyg 2009, 81: 875-881.

26. Cheng PN, Wang RH, Wu IC, Wu JC, Tseng KC, Young KC, Chang TT: Seroprevalence of hepatitis $\mathrm{E}$ virus infection among institutionalized psychiatric patients in Taiwan. J Clin Virol 2007, 38: 44-48.

27. Kumar A, Aggarwal R, Naik SR, Saraswat V, Ghoshal UC, Naik S: Hepatitis E virus is responsible for decompensation of chronic liver disease in an endemic region. Indian J Gastroenterol 2004, 23: 59-62.

doi:10.1186/1743-422X-8-152

Cite this article as: Zhang et al:: Clinical characteristics and risk factors of sporadic Hepatitis E in central China. Virology Journal 2011 8:152.

\section{Submit your next manuscript to BioMed Central and take full advantage of:}

- Convenient online submission

- Thorough peer review

- No space constraints or color figure charges

- Immediate publication on acceptance

- Inclusion in PubMed, CAS, Scopus and Google Scholar

- Research which is freely available for redistribution

Submit your manuscript at www.biomedcentral.com/submit
Ciomed Central 